\title{
Funktionelle Anatomie und Biomechanik des Handgelenkes und distalen Radioulnargelenkes
}

\author{
Johannes Frank, Hans Pralle, Ingo Marzi
}

\section{Zusammenfassung}

Das distale Ende des Radius bildet das anatomische Fundament des Handgelenkes. Die knöchernen Strukturen und Bandverbindungen bilden die Grundlage für die hohe Beweglichkeit und Kraftübertragung von der Hand auf den Unterarm. Das Handgelenk setzt sich proximal aus der radio-carpalen Gelenkfläche, dem distalen Radioulnargelenk und dem triangulären Faserknorpel (TFC) zusammen. Distal wird das Handgelenk von der ersten Handwurzelknochenreihe gebildet, die durch intrinsische Bänder zusammengehalten werden. Die Stabilität des Handgelenkes selbst wird durch die Gelenkkapsel und vor allem die das Gelenk überbrückenden dorsalen und palmaren extrinsischen Bänder gewährleistet. Verletzungen des distalen Radius mit Verkürzung und Verkippung führen regelmäßig zu einer Mitverletzung dieser Strukturen und damit zu funktionellen Defiziten. Diese Verletzungen erfordern häufig eine differenzierte klinische, radiologische oder arthroskopische Diagnostik. Neben einer möglichst anatomischen Wiederherstellung des distalen Radius müssen daher die Verletzungen des distalen Radioulnargelenkes (DRUG), des TFC und der wichtigen Bandstrukturen, vor allem das scapho-lunäre Band, rekonstruiert werden. Das funktionelle Ergebnis ergibt sich daher aus der anatomischen Wiederherstellung des distalen Radius einschließlich seiner unmittelbaren Begleitstrukturen.

\section{Einleitung}

Der distale Radius stellt den Hauptpfeiler des Handgelenkes dar und ist für die Kraftübertragung verantwortlich. Die radiocarpale Gelenkfläche wird nach ulnar hin durch den triangularen Faserknorpel (TFC) komplettiert und ist eng verbunden mit dem distalen Radio-Ulnargelenk (DRUG). Die Verbindung zur Hand selbst erfolgt über starke dorsale und palmare Bandverbindungen zu den Handwurzelknochen. Diese komplexe anatomische Konstellation erlaubt einen hohen Bewegungsgrad sowohl im Handgelenk, als auch die Unterarmrotation. Dieser Aufbau führt jedoch dazu, dass bei Verletzungen des distalen Radius diese anatomischen Strukturen immer mitbeurteilt und therapiert werden müssen. (Abb.1).

OP-JOURNAL 2003; 19: 4-9

(c) Georg Thieme Verlag Stuttgart . New York

\section{Hauptteil}

Das radio-carpale Gelenk ist das zentrale Element des Handgelenkes und bildet dessen Hauptgelenkfläche. Hand und Radius bilden gemeinsam eine Einheit, die gelenkig mit der Ulna verbunden ist und um diese rotiert. Der distale Radius hat 3 konkave Gelenkflächen: 1. Fossa Scaphoidea, 2. Fossa Lunata, 3. Fossa der Articulatio radioulnaris distalis, die sogenannte „Sigmoid Notch“ (Abb.2). Die Fossa scaphoidea hat eine Dreiecksform, deren Spitze den Processus styloideus radii formt. Eine dorso-palmare Crista trennt diese von der kleineren Fossa lunata. Beide Gelenkflächen sind konkav in der anterior/posterioren und radial/ulnaren Ebene. Das distale Radioulnargelenk (DRUG) ist konkav und nach distal, dorsal und palmar scharf begrenzt, nicht jedoch nach proximal.

Der metaphysäre Bereich des Radius beginnt etwa $2 \mathrm{~cm}$ vor der distalen Gelenkfläche und ist gekennzeichnet durch eine Abnahme der Dicke der Kortikalis bei gleichzeitiger Zunahme an spongiösem Knochen. In der frontalen Ebene ist die Radiusgelenkfläche mit einem Winkel von circa 22-23 Grad nach ulnar geneigt und stellt den knöchernen tragenden Anteil des Handgelenksbogens dar (Abb. 3). In der sagittalen Ebene ist die Gelenkfläche des Radius im Durchschnitt zwischen 11 - 12 Grad nach palmar geneigt (Abb.4).

Diese Inklination ist für die korrekte Kraftübertragung zwischen dem distalen Radius und den Handwurzelknochen von erheblicher Bedeutung.

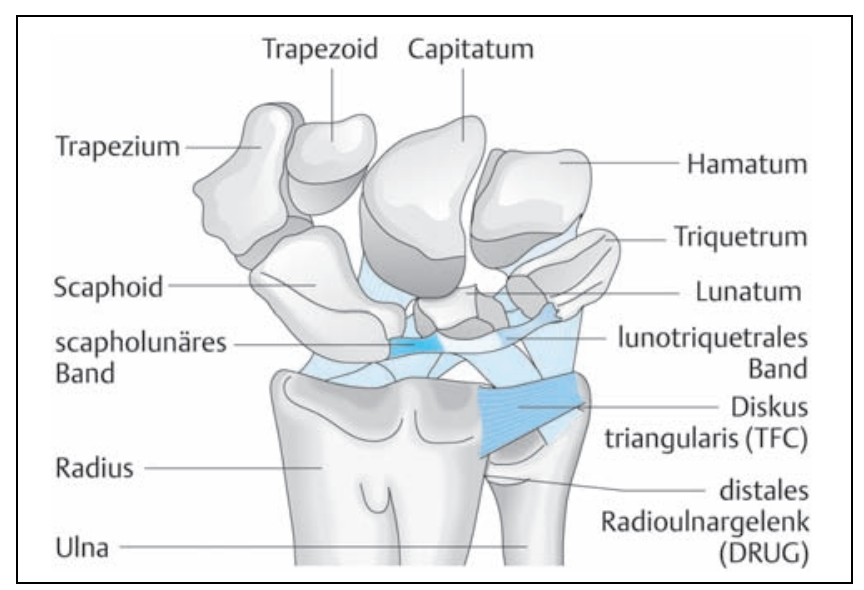

Abb.1 Schematische Darstellung des Handgelenkes mit distaler Ulna und Radius, sowie die angrenzenden Handwurzelknochen. 


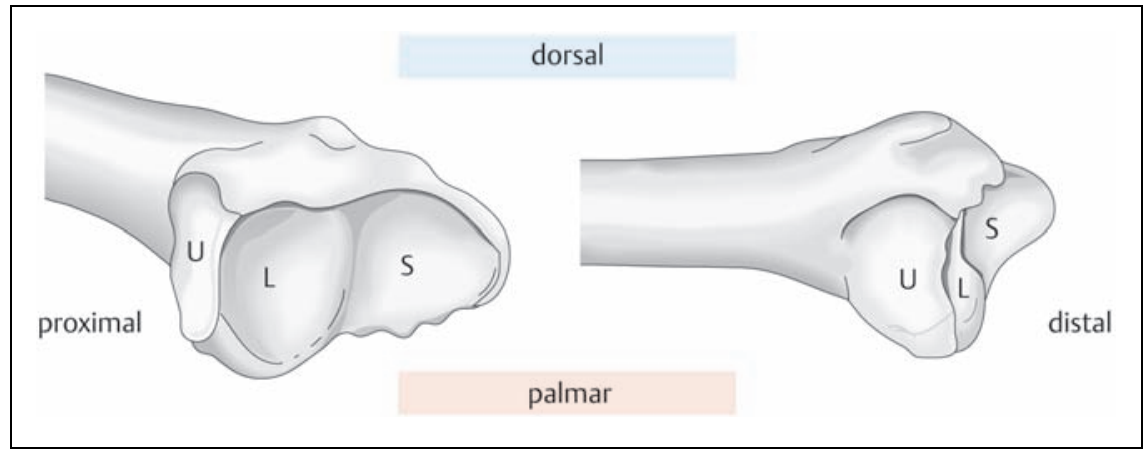

Abb.2 Schematische Darstellung der Anatomie des distalen Radius mit Gelenkflächen. U: UInare Gelenkfläche („Sigmoid notch“) ; L: Fossa lunata; S: Fossa scaphoidea.
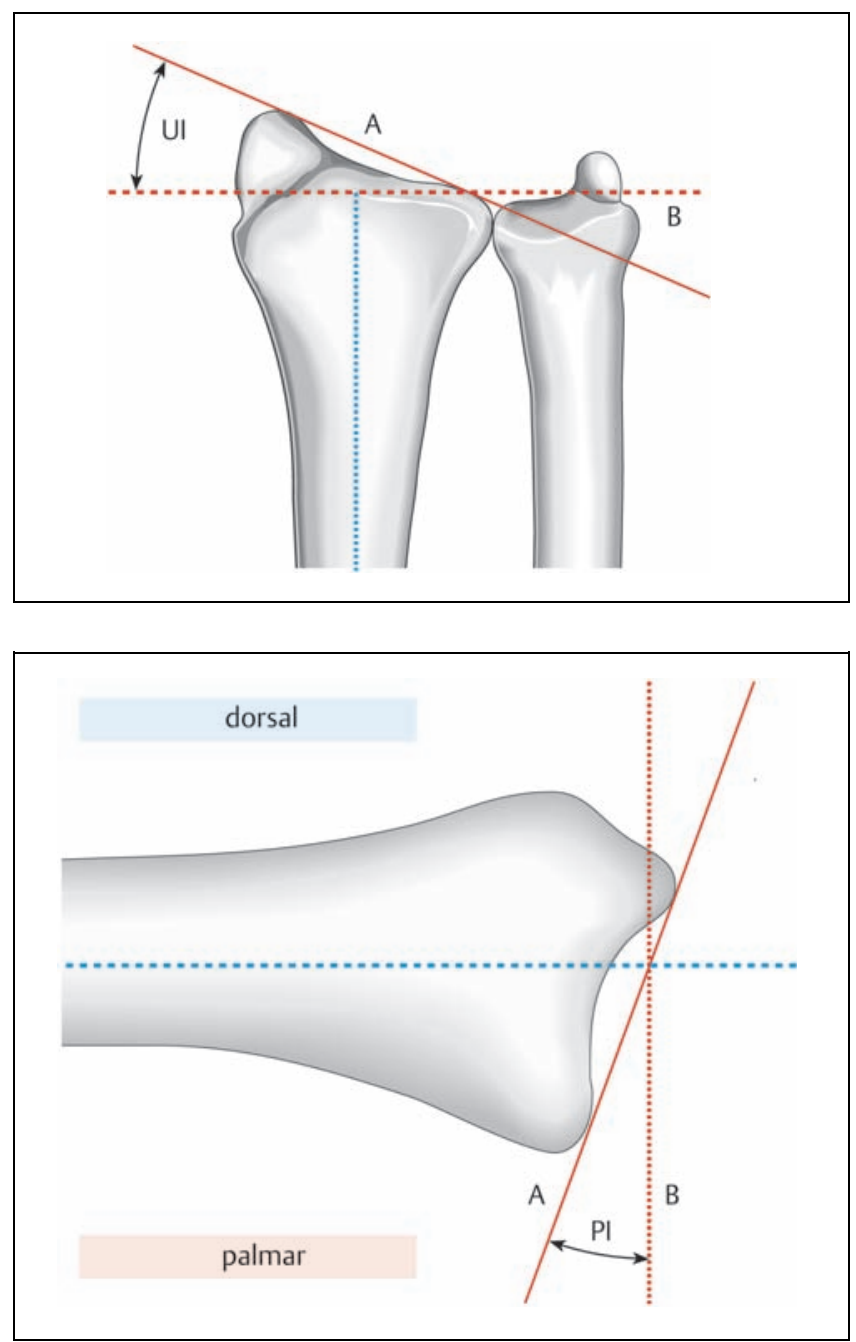

Abb. 4 Bestimmung der Palmarinklination des distalen Radius. Die Palmarinklination wird bestimmt als Winkel (PI) zwischen der Parallelen zur Gelenkfläche $(A)$ und der Senkrechten zur Längsachse des Radius (B). Im Normalfall ca. 12 Grad $(0-$ 28 Grad).

Die Messung der relevanten Gelenkwinkel im AP Röntgenbild und im seitlichen Röntgenbild ist in Abb. $\mathbf{6}$ exemplarisch an einer dislozierten Fraktur mit pathologischen Gelenkwinkeln dargestellt $[2,6]$.

Der palmare Bereich des Radius ist flach und stellt den Ansatzpunkt für wichtige das Handgelenk stabilisierende Band- dem langen Daumenstrecker, als Widerlager dient. Diese Sehne des M. extensor pollicis longus umläuft diese Knochenstruktur, die ihm als Hypomochlion dient.

Das Handgelenk ist ein ausgesprochen bewegliches Gelenk und erlaubt etwa eine Extension/Flexion von 120 Grad und eine Radial-/Ulnardeviation von 50 Grad. Die Rotation des Unterarmes beträgt etwa 150 Grad und erfolgt im distalen Radioulnargelenk durch Rotation von Radius mit Hand um den Ulnakopf, der über die Ulna und die Struktur des Ellenbogengelenkes fixiert ist.

Um einerseits eine uneingeschränkte Unterarmrotation im distalen Radioulnargelenk (DRUG) zu ermöglichen und andererseits die Ulnarabduktion und freie Bewegung im Handgelenk zu gewährleisten ist eine korrekte Länge und Stellung des distalen Radius in Relation zur distalen Ulna von erheblicher Bedeutung.

Die individuelle Längenrelation von distalem Radius zur distalen Ulna unterliegt einer Varianz, die bei der Beurteilung gegebenenfalls unter Vergleich mit der Gegenseite berücksichtigt werden muss. Man geht davon aus, dass Variationen bis zu $4 \mathrm{~mm}$ ohne pathologische Bedeutung sind. Die korrekte Erfassung der radialen Länge und der Ulnarvarianz ist in Abb. 5 dargestellt $[1,2,6]$.

Untersuchungen haben gezeigt, dass etwa $80 \%$ der axialen Belastung über die radiokarpale Gelenkfläche auf den Unterarm übertragen wird. Zwanzig Prozent wird über das ulnare Kompartment weitergeleitet. Damit diese Kräfte übertragen werden können und dennoch der hohe Grad an Beweglichkeit bewahrt werden kann sind komplexe Band- und Weichteilstrukturen zur Sicherung der Stabilität erforderlich. Die Integrität und Stabilität der Bandstrukturen lässt sich am Standard-Röntgenbild nur indirekt beurteilen.

Als gute Orientierung hat sich hier die Verfolgung der 3 von Gilula beschriebenen bogenförmigen Linien bewährt. Diese Linien sollen im Normalfall ohne Versatz oder Unterbrechung den Verlauf der Gelenkflächen der Handwurzelknochen als rundliche Bögen abbilden.

Bei einer Luxation oder Luxationsfraktur sind diese Bögen unterbrochen und weisen auf eine schwerwiegende Verletzung hin (Abb. 7) [4,6]. 


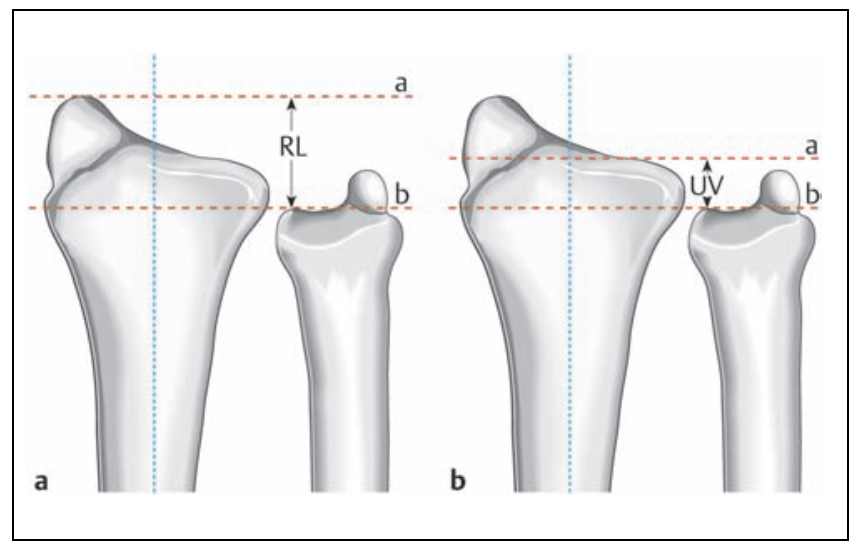

Abb. 5 Schematische Darstellung zur Bestimmung der radialen Länge am distalen Radius und der Ulnarvarianz. A: Die radiale Länge wird im AP-Strahlengang bestimmt und ist der Abstand zwischen der Senkrechten zur Achse des Radius durch die Spitze des Processus styloideus radii (a) und der Senkrechten in Höhe der distalen Gelenkfläche des Ulnakopfes (b). Im Normalfall ca. $12 \mathrm{~mm}(8-18 \mathrm{~mm})$. B: Die Ulnarvarianz wird ermittelt aus dem Abstand zwischen der Parallelen zur Fossa lunata (a) und der zur distalen Gelenkfläche des Ulnakopfes (b). Bei ca. 60\% der Patienten befindet sich die distale Gelenkfläche des Ulnakopfes und der ulnare Rand des Radius auf der gleichen Höhe. Es findet sich eine Variation zwischen +4 und $-4 \mathrm{~mm}$ ohne Pathologie.

Die Stabilität des Handgelenks wird von extrinsischen und intrinsischen Bändern garantiert, die von Radius und Ulna zu den Handwurzelknochen der 1. und 2. Reihe ziehen (Abb.1). Die extrinsischen Bänder überbrücken Radiokarpal-, Ulnokarpal- oder das Interkarpalgelenk (Abb.8). Radiokarpal finden sich hier
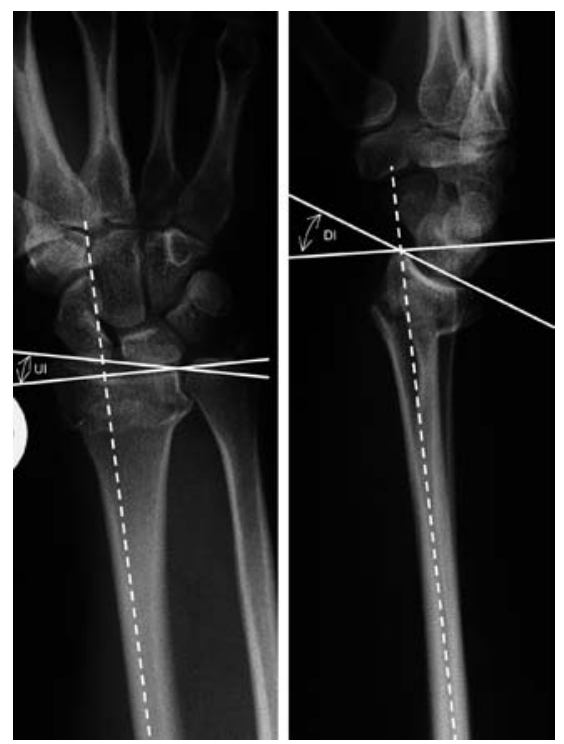

Abb. 6 Röntgenbild mit Darstellung des Handgelenkes und distaler Radiusfraktur. Es zeigt sich im AP-Strahlengang und in der seitlichen Röntgenaufnahme die pathologisch veränderte Ulnarinklination (UI) und Palmarinklination. Es findet sich keine Palmarinklination sondern eine Abkippung nach dorsal (DI). gibt Tab.1. $[4,5,6]$ a

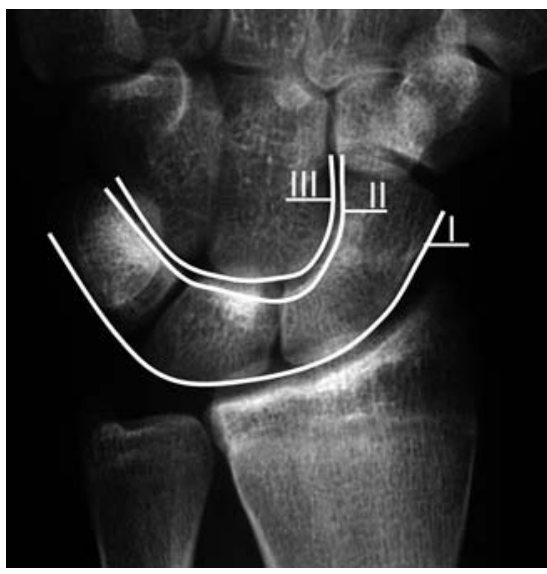

b

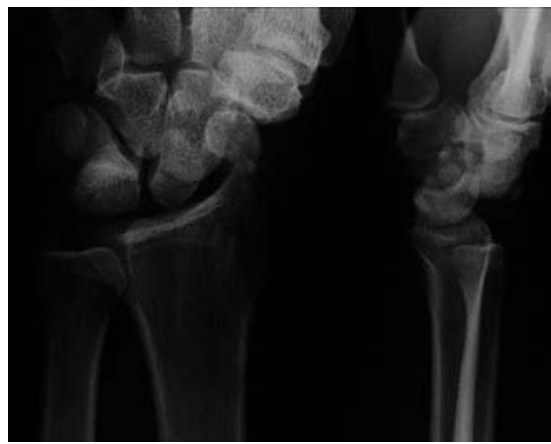

Abb. 7 Hilfreich für die Beurteilung der Stellung der Handwurzelknochen sind die 3 von Gilula beschriebenen Bögen. A: Diese „karpalen Bögen" sollten im Normalfall glatt entlang der Gelenkflächen der Handwurzelknochen verlaufen ohne plötzlichen Sprung oder unterschiedliche Weiten der Gelenkspalten (Bogen I, II u. III). B: Dieser Teil der Abbildung zeigt ein Röntgenbild nach transscaphoidärer perilunärer Luxationsfraktur. Die Bögen sind alle unterbrochen und im seitlichen Strahlengang ist deutlich die Luxationsstellung zwischen Lunatum und Capitatum zu erkennen.

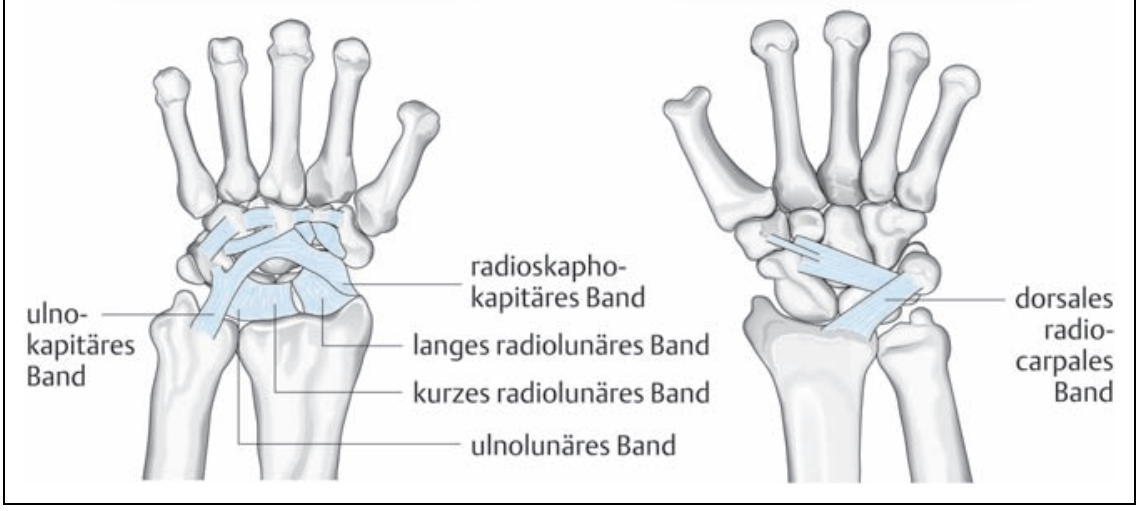

Abb.8 Schemazeichnung der extrinsischen karpalen Bandstrukturen. 
Tab 1 Karpale Bandstrukturen.

\begin{tabular}{cl}
\hline Extrinsische Bänder & Intrinsische Bänder \\
\hline oberflächliche und tiefe Radiokarpale Bänder: & Skapholunäres Interossäres Lig. (SL) \\
$\square$ Radioskaphokapitäres Lig. (RSC) & Lunotriquetrales Lig. (LTq) \\
$\square$ Langes Radiolunäres Lig. (LRL) & Distale Reihe: Interossäre Bänder \\
$\square$ Radioskapholunäres Lig. (RSL) & Palmare interkarpale Bänder: \\
$\square$ Kurzes Radiolunäres Lig. (SRL) & Triquetrohamatokapitäres Lig. (TqHC) \\
$\square$ Dorsales Radiotriquetrales Lig. (RTq) & Skaphokapitäres Lig. (SC) \\
Oberflächliche und Tiefe Ulnokarpale Bänder: & Skaphotrapeziotrapezoidäres Lig. (STT) \\
$\square$ Ulnokapitäres Lig. (UC) & Dorsale Interkarpale Bänder (DIC) \\
$\square$ Ulnotriquetrales Lig. (UTq) & \\
$\square$ Ulnolunäres Lig. (UL) &
\end{tabular}

Tab.2 Arthroskopische Klassifikation der Verletzungen des scapholunären und lunotriquetralen Bandes. [5]

\begin{tabular}{ll} 
Schweregrad & Arthroskopischer Befund \\
\hline Grad I & Ligamentäre Einblutung (radiocarpal) \\
& keine Inkongruenz midcarpal \\
Grad II & Ligamentäre Einblutung (radiocarpal) \\
& Inkongruenz midcarpal, Taststab passt nicht interossär \\
Grad III & Inkongruenz (radiocarpal u. midcarpal) \\
& Taststab passt interossär \\
Grad IV & Inkongruenz (radiocarpal u. midcarpal) \\
& Instabilität bei Manipulation \\
& 2,7 mm Arthroskop passt interossär
\end{tabular}

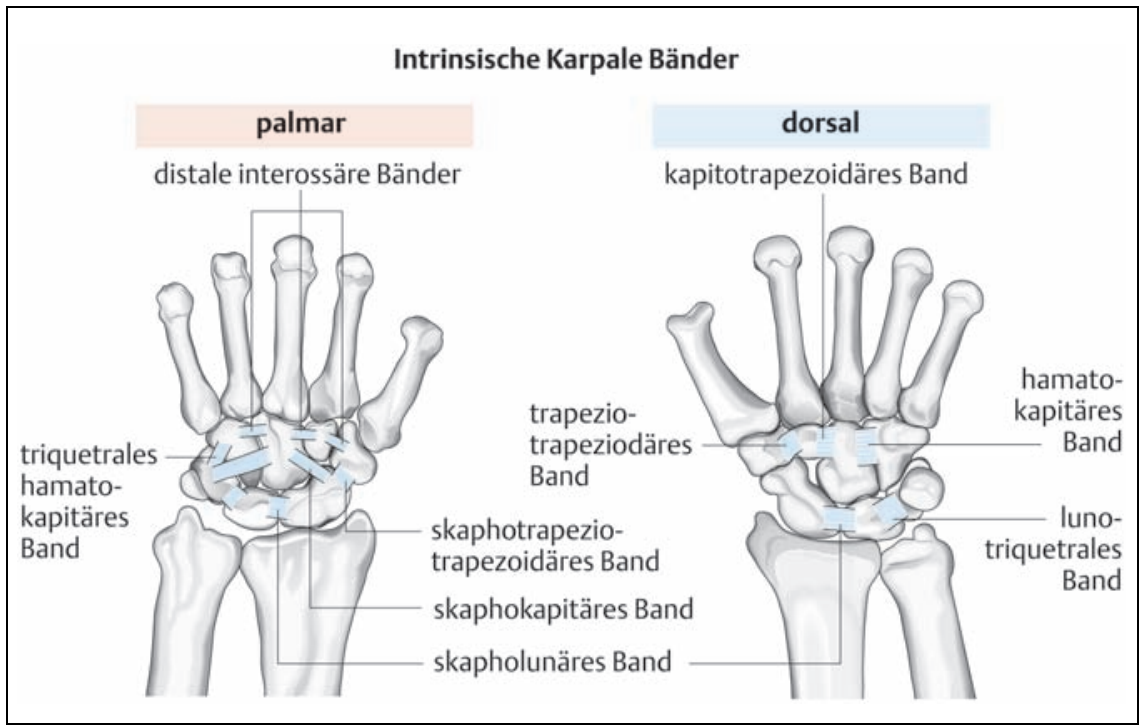

Abb. 9 Schemazeichnung der intrinsischen karpalen Bandstrukturen.

Von erheblicher Bedeutung sind die Rupturen des Scapho-lunären (SL) Bandes, die insbesondere bei B1 und C Frakturen des Radius beobachtet werden.

Ein Übersehen dieser Bandruptur resultiert in einem zunehmenden Kollaps der ersten Handwurzelreihe mit erhebli-
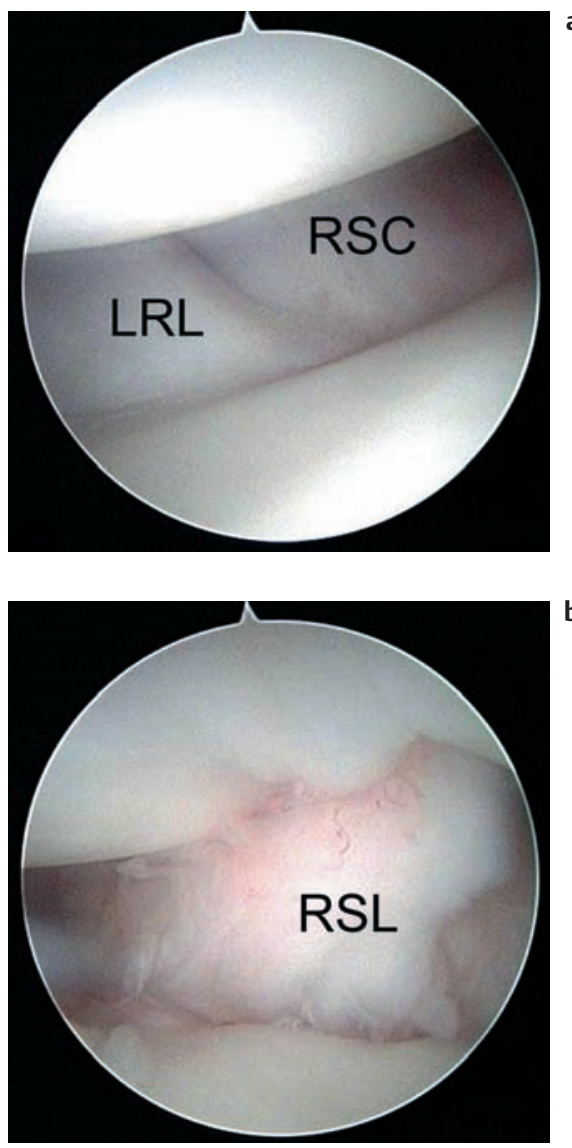

Abb.10 Arthroskopisches Bild der karpalen Bandstrukturen. RSC: Radioskaphokapitäres Band; LRL: Langes Radiolunäres Band; RSL: Radioskapholunäres Band.

aufnahmen in Radial- und Ulnarduktion oder durch MRT gesichert werden. Diese Bandstrukturen, sind jedoch gut im Rahmen einer Handgelenksarthroskopie zu visualisieren (Abb.10). Dabei kann auch deren Stabilität mit dem Tasthaken direkt überprüft und klassifiziert werden, was insbesondere für das scapholunäre Band von erheblicher Bedeutung ist (Tab.2). Die Transfixation und/oder Naht des SL-Bandes, aber auch des LTBandes, ist für die stabile Ausheilung von eminenter Bedeutung $[3,9,10]$.

Der Aufbau der proximalen Handgelenksfläche stellt sich demnach als eine komplexe Konstruktion dar. Wie bei der Aufsicht von distal (Abb.11) erkennbar ist, wird die in Fossa scaphoidea und Fossa lunata unterteilte Gelenkfläche des Radius von den dorsalen und palmaren Bandstrukturen überbrückt. Die extrinsischen Bandstrukturen umgreifen jedoch auch das ulnare Kompartment. Im Zentrum des ulnaren Kompartment befindet sich der trianguläre Faserknorpel (TFC bzw. Diskus triangularis) der die Gelenkfläche 

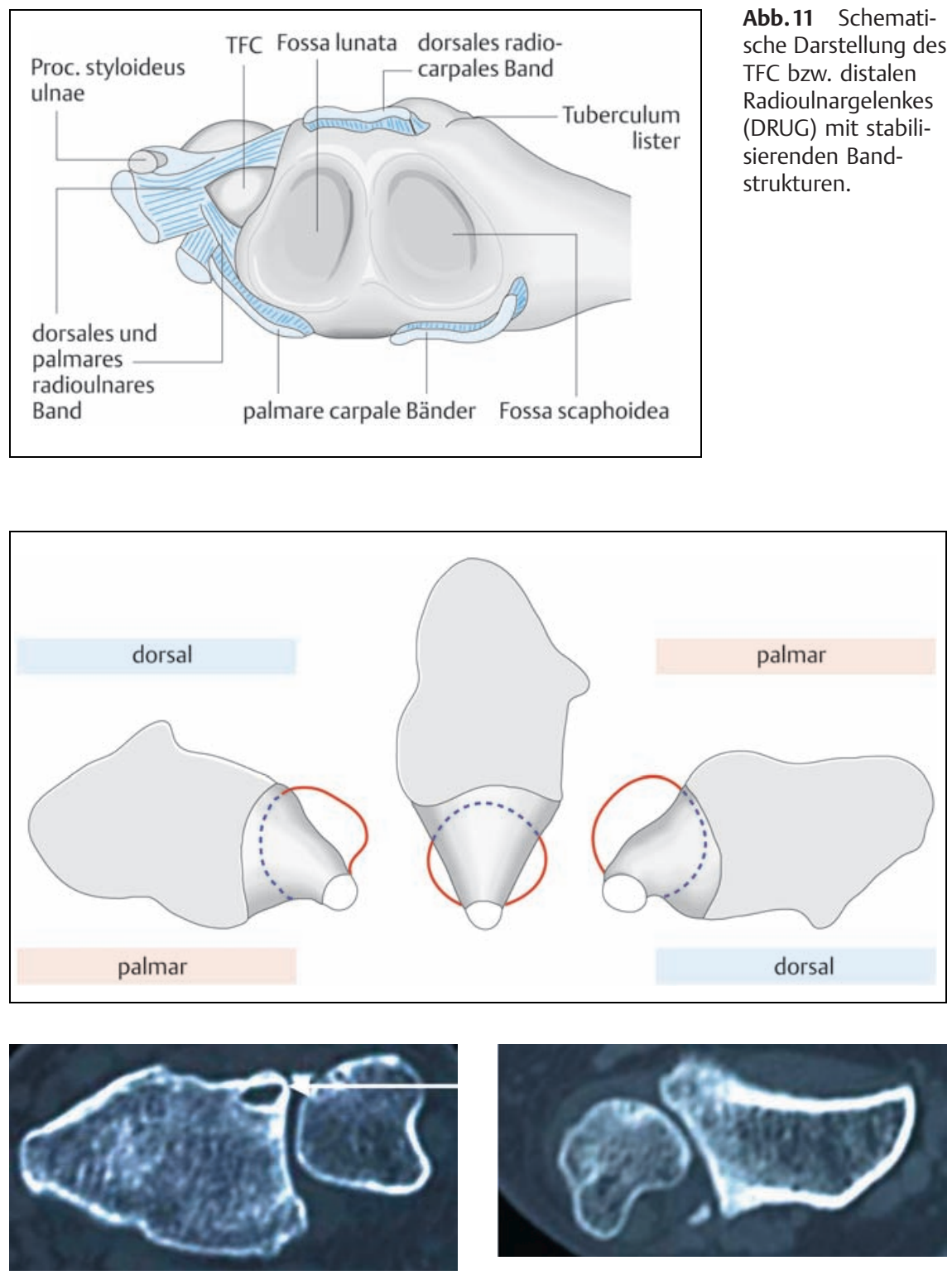

Abb.12 Rolle des TFC bei Pronation u. Supination als Stabilisator des distalen Radioulnargelenks. Zusätzlich dargestellt sind computertomographische Bilder bei posttraumatischer Arthrose des distalen Radioulnargelenkes in den verschiedenen Positionen (Pronation, Supination). Eine geringgradige Verschiebung des Radius nach palmar in Relation zur Ulna ist erkennbar als Ausdruck einer Instabilität (Pfeil).

dann nach ulnar komplettiert. Die distale Ulna hat somit keinen direkten Kontakt zur proximalen Handwurzelreihe. Der dorsale und palmare Anteil des TFC werden als dorsales und palmares radioulnares Band bezeichnet und stabilisieren das distale Radioulnargelenk bei den Umwendbewegungen des Unterarmes. Das distale Radioulnargelenk (DRUG) wird zusätzlich durch folgende Faktoren stabilisiert: die interossäre Membran zwischen Radius und Ulna, den Musculus pronator quadratus, die Sehnen und Sehnenscheiden des M. extensor carpi ulnaris und des M. flexor carpi ulnaris. Post-
Abb.11 Schematische Darstellung des TFC bzw. distalen Radioulnargelenkes (DRUG) mit stabilisierenden Bandstrukturen. throse im DRUG führen. Die Diagnose kann gut mittels Computertomographie dargestellt werden. Gleichzeitig können Instabilitäten des DRUG bei den Verschiedenen Positionen des Handgelenkes (Pronation / Supination) analysiert werden. (Abb.12) [6,7].

Der Diskus triangularis, kurz TFC (= triangular fibrocartilage) genannt, bildet gemeinsam mit den oben dargestell-

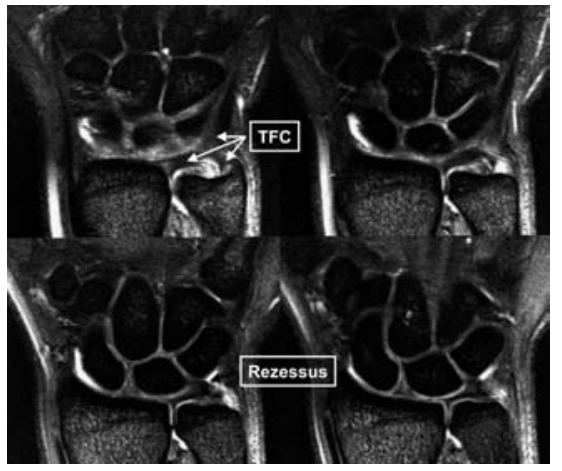

Abb.13 Kernspintomographisches Bild mit Handwurzelknochen und Diskus triangularis (TFC). Die Fasern des TFC die zu Prozessus styloideus ulnae bzw. den Handwurzelknochen verlaufen sind deutlich von den Fasern zu differenzieren, die zur Inzisur an der Basis des Prozessus styloideus ziehen. Die kleine volar gelengene Öffnung im TFC (Rezessus) ist ebenfalls zu erkennen und darf nicht mit einer Ruptur verwechselt werden.

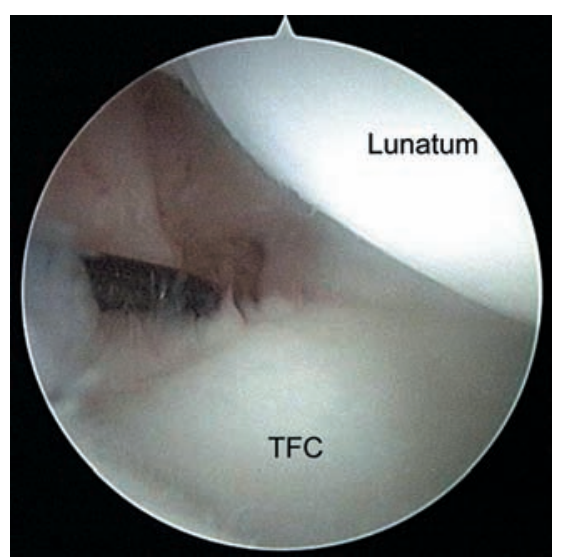

Abb.14 Arthroskopisches Bild des TFC in der Aufsicht. Der Tasthaken verschwindet in dem Rezessus (Normalbefund).

ten Bandstrukturen den Hauptanteil des TFCC (triangular fibrocartilage complex). Dieser Bandkomplex stabilisiert neben dem DRUG vor allem das ulnarseitige Handgelenk. Der TFC inseriert an der distalen ulnarseitigen Gelenkfläche des Radius und zieht über die distale Ulna sowohl zum Processus styloideus ulnae als auch der Fovea des Ulnakopfes (Abb.13 und Abb.14).
Der Verdacht auf eine Läsion des TFC ergibt sich klinisch bei folgenden Befunden: Instabilität des distalen Radioulnargelenkes, meist durch schmerzhafte federnde Prominenz der distalen Ulna nach dorsal; bei schmerzhafter Ulnarbewegung (ulnares Impingement); bei Abrissfrakturen des Proc. styl. ulnae und bei Galeazzi-Verletzungen. 
Tab. 3 Klassifikation der traumatischen und degenerativen TFCC (triangular fibrocartilage complex) Veränderungen. [8] TFC: triangular fibrocartilage; LT: Lunotriquetral

Traumatisch
Klassifikation I
IA: Zentrale Perforation des TFC
IB: ulnarseitige Avulsion mit/ohne dist.
Ulnafraktur
IC: distale carpale Avulsion

ID: radialseitige Avulsion mit/ohne Fraktur der „Sigmoid Notch“

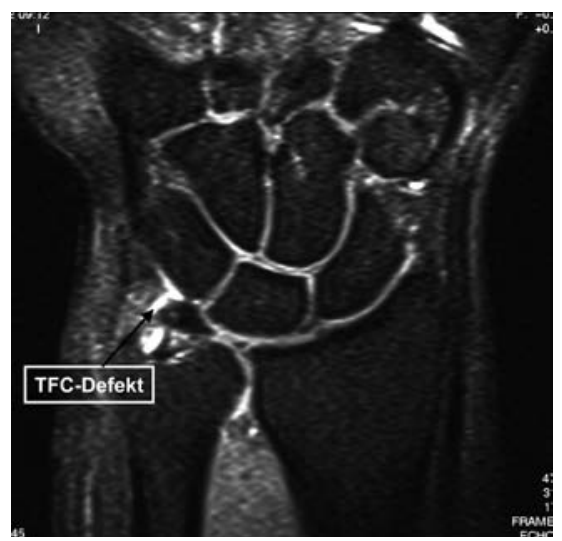

Abb.15 Kernspintomographische Darstellung einer TFC-Ruptur.

Zur Sicherung der Diagnose eignen sich vor allem die Magnetresonanztomographie (MRT) und die Handgelenksarthroskopie, die eine weitere Klassifizierung der TFC-Veränderungen ermöglicht (Tab.3). Als nicht-invasives Verfahren hat sich in den letzten Jahren die hochauflösende MRT als wertvoll erwiesen. Hierbei können die verschiedenen TFCVerletzungen, Rupturen der extrinsischen und intrinsischen Bänder, aber auch okkulte Frakturen des Radius und der Handwurzel nachgewiesen werden. Eine ulnare Ruptur des TFC ist in Abb.15 dargestellt. Als Therapie bietet sich in diesen Fällen die Arthroskopie des Handgelenks an, wobei bei zentralen Defekten ein Debridement und bei ulnaren Rupturen eine

\section{Degenerativ}

Klassifikation II

Stadium 1: TFCC - Verschleiß

Stadium 2: TFCC-Verschleiß u. Lunatumu./od. Ulna- Chondromalazie

Stadium 3: TFCC-Perforation u. Lunatumu./od. Ulna- Chondromalazie

Stadium 4: TFCC-Perforation u. Lunatumu./od. Ulna- Chondromalazie u. LT-Bandperforation

Stadium 5: TFCC-Perforation u. Lunatumu./od. Ulna- Chondromalazie u. LT-Bandperforation u. ulnocarpale Arthrose

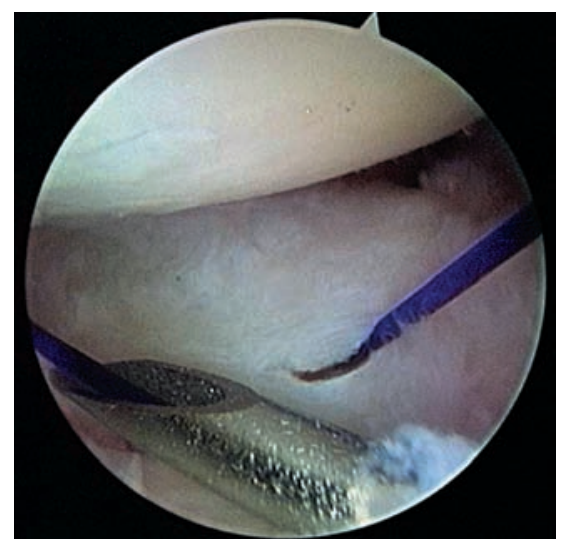

Abb.16 Arthroskopisches Bild einer ulnarseitigen TFC-Ruptur (IB). Auf der Abbildung sind die Fäden bzw. die Kanüle sichtbar mit denen die arthroskopische Refixation erfolgte.

arthroskopische Refixation indiziert ist (Abb. 16). [6, 8, 9,10].

\section{Schlussfolgerung}

Bei allen Verletzungen des distalen Radius müssen immer die eng verbundenen anatomischen Strukturen und funktionellen Abläufe berücksichtigt werden. Eine möglichst exakte Wiederherstellung der Länge, Achse und Neigung des distalen Radius ist Vorraussetzung für eine optimale Funktion des Handgelenkes. Verletzungen des distalen Radioulnargelenkes sowie der wichtigen Bandverbindungen des Handgelenkes und der Handwurzelknochen müssen mittels klinischer Untersuchung, radiologischen Funktions- aufnahmen, MRT oder Handgelenksarthroskopie diagnostiziert werden. Die Versorgung dieser Begleitverletzungen ist für das subjektive und objektive Behandlungsergebnis distaler Radiusfrakturen von entscheidender Bedeutung.

\section{Literatur}

${ }^{1}$ Aro HT, Koivunen T. Minor axial shortening of the radius affects outcome of Colles' fracture treatment. J Hand Surg [Am] 1991; 16: $392-398$

${ }^{2}$ Fernandez DL, Jupiter JB. Fractures of the distal radius. A practical approach to management. New York, Berlin, Heidelberg: Springer-Verlag, 1996

${ }^{3}$ Gabl M, Lener M, Pechlaner S, Judmaier W. The role of dynamic magnetic resonance imaging in the detection of lesions of the ulnocarpal complex. J Hand Surg [Br] 1996; 21: $311-314$

${ }^{4}$ Garcia-Elias M. Kinetic analysis of carpal stability during grip. Hand Clin 1997; 13: 151 158

${ }^{5}$ Geissler WB, Freeland AE, Savoie FH, McIntyre LW, Whipple TL. Intracarpal soft-tissue lesions associated with an intra-articular fracture of the distal end of the radius. Bone Joint Surg [Am] 1996; 78: 357-365

${ }^{6}$ Green DP, Hotchkiss RN, Pederson WC. Greenÿs Operative Hand Surgery. New York, Edinburgh, London, Philadelphia, San Francisco: Churchill Livingstone, 1999

${ }^{7}$ Jaffe R, Chidgey LK, LaStayo PC. The distal radioulnar joint: anatomy and management of disorders. J Hand Ther 1996; 9: 129-138

${ }^{8}$ Palmer AK. Triangular fibrocartilage complex lesions: a classification. J Hand Surg [Am] 1989; 14: 594-606

${ }^{9}$ Rose S, Frank J , Marzi I. Diagnostische und therapeutische Bedeutung der Arthroskopie bei der distalen Radiusfraktur. Zentralbl Chir 1999; 124: 984-992

${ }^{10}$ Urbschat K, Frank J, Marzi I, Schneider G, Roth R. Wertigkeit der MRT in der Diagnostik posttraumatischer Handgelenksbeschwerden. Zentralbl Chir 1999; 124 977-983

\section{Dr. med. Johannes Frank}

Oberarzt

\section{Hans Pralle}

Assistenzarzt

Prof. Dr. med. Ingo Marzi

Direktor der Klinik

Klinik für Unfall-, Hand- und Wiederherstellungschirurgie Klinikum der Johann Wolfgang Goethe-Universität 60590 Frankfurt/Main 7 